\title{
Terminology for Membrane Distillation
}

\author{
K. SMOLDERS* and A.C.M. FRANKEN \\ Faculty of Chemical Technology, Twente University of Technology, P.O. Box 217, \\ 7500 AE Enschede (The Netherlands)
}

(Received May 25, 1989)

\section{INTRODUCTION}

One of the subjects of the "Round Table" at the "Workshop on Membrane Distillation" in Rome on May 5, 1986 was nomenclature. The best example for the need of a more uniform language is the name of the process itself. In Rome the following names were used by the authors present: membrane distillation, Trans Membrane Distillation, thermo-pervaporation, pervaporation and membrane evaporation.

At this workshop a committee was formed with the task of preparing a terminology for membrane distillation. The committee consists of the following members: V. Calabro (Universita della Calabria, Calabria, Italy), A.C.M. Franken (Twente University of Technology, Enschede, The Netherlands), S. Kimura (University of Tokyo, Tokyo, Japan), S. Ripperger [Enka (Membrana), Wuppertal, F.R.G.], G. Sarti (Universita di Bologna, Bologna, Italy), R. Schofield (University of New South Wales, Kensington, N.S.W., Australia).

In this article terms, definitions and symbols, which are used in the field of membrane distillation, are defined. The basis for this document is formed by the terminology for pressure driven membrane operations [1]; whereever this is relevant the terms in this document are defined in the same way.

\section{NAME OF THE MEMBRANE OPERATION}

The most suitable name for this operation is membrane distillation. This name has the advantage that it is already used by most authors, that it has no commercial ties with a company and that it cannot be confused with other membrane processes.

\footnotetext{
*Author to whom all correspondence should be addressed.
} 
For the same reasons as mentioned above, the names Trans Membrane Distillation (the commercial name for the Enka process), pervaporation and thermopervaporation (already used for other membrane operations [2]) should not be used. Introducing a new name, like membrane evaporation, has the disadvantage that both author and reader will have adjustment problems, that may result in an even greater confusion of tongues.

The name pervaporation is often used by Japanese authors for two different processes: the process of membrane distillation and the process of pervaporation. In our opinion an essential difference exists between these processes. According to our definition (see later in this article), membrane distillation is a process in which the membrane itself has no influence on the vapour-liquid equilibrium of the liquids to be separated. On the other hand, pervaporation is a process in which liquid diffuses through a membrane and evaporates at the permeate side of the membrane. The separation characteristics of the pervaporation process are determined by sorption into an diffusion through the membrane.

\section{DESCRIPTION OF THE MEMBRANE OPERATION}

\section{Characteristics of membrane distillation}

The name "membrane distillation" should be applied for membrane operations having the following characteristics:

- the membrane should be porous;

- the membrane should not be wetted by the process liquids;

- no capillary condensation should take place inside the pores of the membrane;

- only vapour should be transported through the pores of the porous membrane;

- the membrane must not alter the vapour-liquid equilibrium of the different components in the process liquids;

- at least one side of the membrane should be in direct contact with the process liquid;

- for each component the driving force of this membrane operation is a partial pressure gradient in the vapour phase.

\section{Different embodiments of membrane distillation}

Many different embodiments of membrane distillation can be found in the literature. For instance, Enka uses a membrane operation in which the liquid on both sides of the membrane is in direct contact with the membrane [3], whereas on the other hand the Swedish National Development Company uses a system in which the vapour is condensed against a cooling-plate [4].

For the different embodiments of membrane distillation the following terms are defined: 
- direct-contact membrane distillation for a system in which the liquid on both sides of the membrane is in direct contact with the membrane and in which the liquid on the downstream side is used as the condensing medium (Fig. 1). - gas-gap membrane distillation for a system in which the vapour on the downstream side is condensed against a cooling surface and in which the condensed liquid on the downstream side does not have to be in contact with the membrane (Fig. 2). In this configuration the condensation of the permeate takes place inside the module.

- low pressure membrane distillation. In this system a low pressure is applied downstream and the condensation of the permeate takes place outside the module. An other term that can be used in this case is: "vacuum membrane

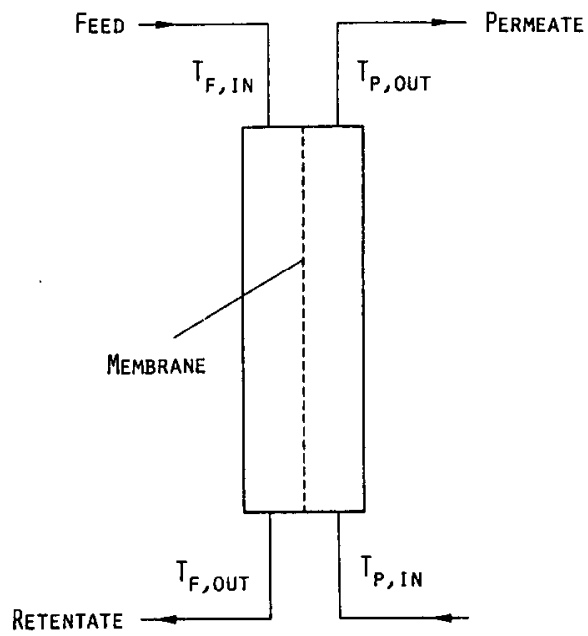

Fig. 1. Direct-contact membrane distillation.

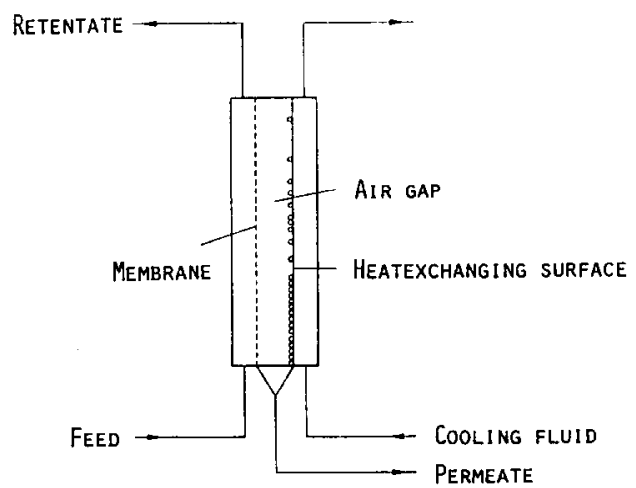

Fig. 2. Gas-gap membrane distillation. 
distillation", but this term is in fact more limiting than low pressure membrane distillation.

- sweeping gas membrane distillation. In this system a sweeping gas (e.g., nitrogen) is applied downstream and the condensation of the permeate takes place outside the module.

Note. If the term "membrane distillation" is used without any further specification, then this term applies to the "direct-contact" system. To avoid complications, it is better to use the full term "direct-contact membrane distillation".

Example. The system of Enka should be called "direct-contact membrane distillation" and the system of the Swedish National Development Company should be called "gas-gap membrane distillation".

\section{MEMBRANE CHARACTERISTICS}

The membranes, used in membrane distillation, should be characterized by the following membrane (performance) parameters: (a) (polymer) material, (b) thickness of the membrane, (c) porosity of the membrane, (d) nominal pore size, (e) liquid-entry-pressure of water.

\section{(a) (Polymer) material}

The material of which the membrane is made is the most important parameter. At this moment membranes for membrane distillation are all made of polymers, but as one should not exclude other type of materials the term polymer is placed between brackets.

(b) Thickness of the membrane

This parameter is of importance because it gives information on both the mechanical strength of the membrane and the fluxes to be expected.

\section{(c) Porosity of the membrane}

The porosity of the membrane is defined as the volume of the pores divided by the total volume of the membrane; the symbol for the porosity is $\epsilon$. A method for the determination of the porosity of (hydrophobic) membranes is suggested in the Appendix.

(d) Nominal pore size

Nominal pore size is important as it can lead to a first approximation of the fluxes to be expected. "Nominal" pore size is a blanket term incompassing pore 
sizes estimated from bubble-point tests, gas permeation experiments, or any other convenient technique, and is usually quoted by the membrane manufacturer. Despite the approximate nature of nominal pore size, it conveys useful information, which can be used to make an approximate calculation of the fluxes to be expected.

\section{(e) Liquid-entry-pressure of water}

The liquid-entry-pressure of water (sometimes faulty called "wetting pressure") is the pressure ( $\mathrm{Pa}$ ) that must be applied onto pure water before it penetrates into a non-wetted (dry) membrane; the symbol for the liquid-entrypressure of water is $\mathrm{LEP}_{w}$. A method for the determination of $\mathrm{LEP}_{w}$ of hydrophobic membranes is suggested in the Appendix.

These five characteristics are suggested, because they give a visual $(a, b)$ and mechanical $(\mathrm{a}, \mathrm{b}, \mathrm{c})$ picture of the membrane, while indicating the suitability for membrane distillation (e) and the fluxes to be expected (b, c, d).

As additional information the following membrane characteristics can be given: (f) IPA bubble point, (g) maximum pore size, (h) pore size distribution, (i) pore size morphology, $(j)$ temperature stability, $(k)$ chemical resistance.

\section{(f) IPA bubble point}

The IPA (isopropylalcohol) bubble point can be measured according to a standard test method as described in ASTM F-316. This method employs a procedure for determining the maximum pore size and the pore size distribution of a membrane filter by measuring the initial bubble point and gas flow versus pressure through a liquid wet filter. The only difference between the "IPA bubble point method" and ASTM F-316 is that IPA is used as the wetting liquid instead of water.

(g) Maximum pore size

The maximum pore size can be calculated by substituting the IPA bubble point pressure into the following formula:

pore size $=\frac{4 B \gamma}{p}$

in which $B=$ pore size morphology constant, $\gamma=$ surface tension of IPA, $p=$ bubble point pressure. The pore size morphology constant $B$ is 1 for a circular pore and less than 1 for an elliptical or irregularly shaped pore [7]. Because most pores are not circular, the use of the terms "pore diameter" and "pore radius" is misleading. Unless membrane with circular pores are used, the term "pore size" is recommended. 
Note. Because the "pore size morphology constant $B$ " is not known in most cases, it is recommended that the "IPA bubble point pressure" is given next to the value of the maximum pore size.

(h) Pore size distribution

The pore size distribution can be measured by means of the standard test method as described in ASTM F-316. A short description of this method is given in $(f)$.

(i) Pore size morphology

The pore size morphology is closely related to the calculation of the "maximum pore size" (g) through the "pore size morphology constant $B$ ". Therefore, it should be given if the pores are, for instance circular, elliptical or rectangular.

\section{(j) Temperature stability}

The long term stability of a membrane to extreme temperatures should be given here.

\section{(k) Chemical resistance}

The chemical resistance to solvents, acids and bases is important, especially if the membranes have to be cleaned.

\section{PROCESS CHARACTERISTICS}

The efficiency of a membrane distillation operation can be characterized in many different ways and depends on many different parameters, such as membrane characteristics, module design, hydrodynamic conditions, temperature level, etc.

The following parameters are defined to characterize a membrane distillation operation and a membrane distillation process: (a) evaporation efficiency, EE; (b) process efficiency, PE; (c) concentration factor, CF; (d) temperature polarization coefficient, TPC.

(a) Evaporation efficiency, EE

This parameter is defined to characterize the efficiency of a membrane distillation operation. The evaporation efficiency $\mathrm{EE}$ is defined as: 
$\mathrm{EE}=\frac{\text { part of the heat which contributes to evaporation }}{\text { total heat input in the module }}$

Besides evaporation also a certain heat transfer due to conduction takes place. Therefore, $\mathrm{EE}$ is always lower than 1 . The value of $\mathrm{EE}$ can be calculated very easily. The "part of the heat which contributes to evaporation" can be calculated by multiplying the measured flux $J$ by the heat of evaporation $\Delta H_{\text {vap }}$ and the membrane area $A$. The "total heat input into the module" can be calculated from a measurement of the caloric value of the incoming and the outgoing feed stream.

(b) Process efficiency, $P E$

This parameter is used to characterize the efficiency of a membrane distillation process (this is a process in which the membrane distillation operation is the most important unit operation). The process efficiency $\mathrm{PE}$ is defined as:

$\mathrm{PE}=\frac{\text { heat which contributes to the evaporation of the distillate }}{\text { total heat input of the process }}$

If the process only consists of a membrane distillation operation then the evaporation efficiency EE is equal to the process efficiency PE. In most cases a heat recovery is advantageously. An example of a membrane distillation process with heat recovery is given in Fig. 3.

(c) Concentration factor, $\mathrm{CF}$

The concentration factor CF is defined as the degree of increasing the concentration of a component in a membrane operation. CF can be calculated by dividing the concentration of the retentate $\left(C_{r}\right)$ by the concentration of the

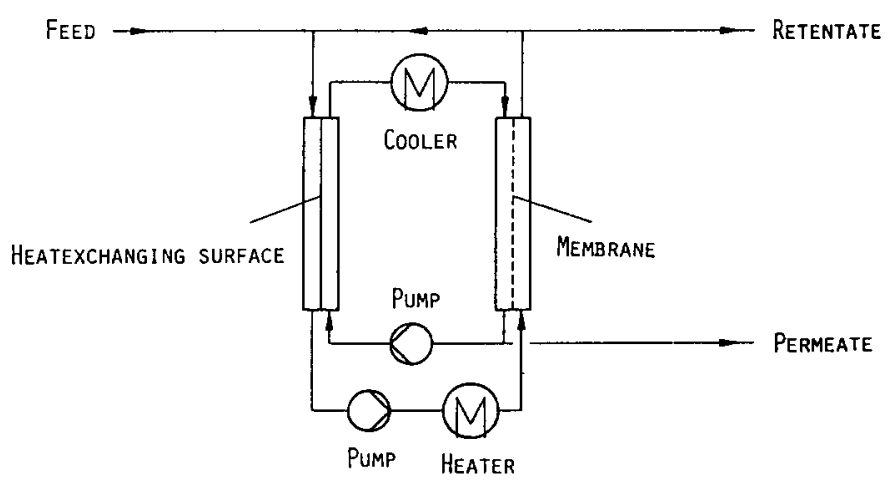

Fig. 3. Membrane distillation process with heat recovery. 


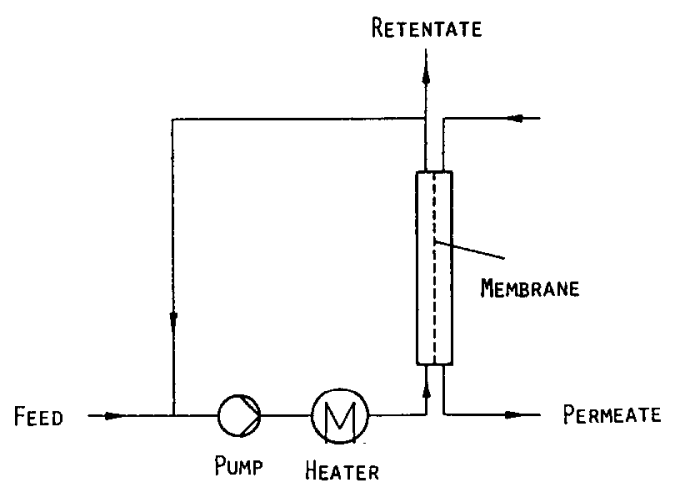

Fig. 4. Membrane distillation process with recirculation of the feed.

feed $\left(C_{\mathrm{f}}\right)$. In formula: $\mathrm{CF}=C_{\mathrm{r}} / C_{\mathrm{f}}$. This term is defined in the same way as for pressure driven membrane operations [1].

Due to the low concentration of the solution by one passage of a membrane distillation module, the solution has to be circulated if a concentration of the feed is required (this situation is shown in Fig. 4).

(d) Temperature polarization coefficient, TPC

Another phenomenon which occurs in a membrane distillation operation is "temperature polarization". Although this term has several disadvantages (its effect cannot be measured directly and the term itself is physically not correct), it is used very often in scientific literature. The value of TPC is given by the temperature difference between the evaporation surface and the condensation surface divided by the temperature difference between the bulk of the feed and the bulk of the permeate.

For direct contact membrane distillation the evaporation surface is formed by the feed-membrane interface and the condensation surface is formed by the permeate-membrane interface. In formula:

$\mathrm{TPC}=\frac{T_{\mathrm{fm}}-T_{\mathrm{pm}}}{T_{\mathrm{fb}}-T_{\mathrm{pb}}}$

It must be stated again that the temperatures at the evaporation (respectively condensation) surface cannot be measured directly. In fact they can only be calculated in special cases when the hydrodynamic conditions on both sides of the membrane are known. 


\section{DEFINITIONS}

In this section a summary is given of the terms that are used in membrane distillation. Whereever this is relevant, the terms are defined in the same way as for pressure driven processes [1].

Bubble point pressure. Pressure at which a continuous stream of gas bubbles is pressed through a liquid wet filter.

Bulk temperature $T_{\mathrm{b}}$. Temperature that exists in the bulk phase; in practice this temperature is equal to the measured temperature.

Circulation loop. A section of a membrane plant containing one or more circulation pumps ensuring adequate cross-flow velocity of the fluid over the membrane (an example is given in Fig. 4).

Concentration factor, $C F$. The degree of increasing the concentration of a component in a membrane operation; $\mathrm{CF}=C_{\mathrm{r}} / C_{\mathrm{f}}$.

Cross-flow velocity, $u$. The velocity of a fluid flowing parallel to the membrane (also called tangential velocity).

Direct-contact membrane distillation. See Different embodiments of membrane distillation.

Evaporation efficiency, EE. See (a) in Process characteristics.

Feed. The fluid entering a membrane module or plant.

Flux, $J$. Amount of permeate, or of any component in the permeate, that is transported through a membrane per unit of membrane area and per unit of time.

Fouling. The deposition of material on the membrane surface and/or in its pores, leading to a change in the membrane performance.

Gas-gap membrane distillation. See Different embodiments of membrane distillation.

Liquid-entry-pressure, LEP. Pressure at which the liquid penetrates into a porous membrane (old term to be replaced by "wetting pressure").

Module. The smallest practical unit containing one or more membranes and supporting structures (old terms to be replaced are permeator, membrane element).

Permeate. The portion of the feed passing through the membrane. Distillate can also be used as a term to describe the "permeate" of membrane distillation, but it is better to use "permeate" because it is commonly used in membrane literature.

Pore size. Openings in a membrane; this term is preferred to "pore diameter" and "pore radius", because all pore shapes can be described by this term.

Porosity. The porosity is defined as the volume of gas that is trapped inside a membrane divided by the total volume of the membrane; a practical definition is given in Appendix 1.

Process efficiency, PE. See (b) in Process characteristics. 
Retentate. The portion of the feed not passing through the membrane (old term: concentrate).

Retention. The ability of a membrane to hinder a component from passing through it or to retain a component in the fluid.

Retention coefficient, $R$. The degree of separation of a certain component from the solvent by the membrane under defined operating conditions; $R=1-\mathrm{C}_{\mathrm{p}} / C_{\mathrm{r}}$. This term should be used if a solution of a solute (e.g., salt) in a solvent (e.g., water) is treated by membrane distillation.

Selectivity, $\alpha$. This term is to be defined as:

$\alpha=\frac{(w t . \% \mathrm{~A} / \mathrm{wt} . \% \mathrm{~B}) \text { in permeate }}{(\mathrm{wt} . \% \mathrm{~A} / \mathrm{wt} . \% \mathrm{~B}) \text { in feed }}$

This term should be used as both components in the membrane distillation systems are volatile.

Tangential velocity, $u$. See Cross-flow velocity.

Temperature polarization coefficient, TPC. See Process characteristics.

\section{SYMBOLS AND UNITS}

It is very difficult to define symbols in such a way that both author and reader are pleased with it. The discussions between the members of the nomenclature committee concentrated on the point of the symbols. The discussions about the definition of symbols took about $80 \%$ of our discussion time, indicating the difficulty of this matter.

To define the symbols the committee has used the following starting-points:

(1) The symbols must be logic and clear to both author and reader.

(2) The symbols must be in agreement with the existing membrane literature. The report on the terminology for pressure driven membrane operations [1] is used as a basis for the definition of the symbols.

(3) The symbols must be in agreement with the literature on heat transfer. The symbols used in the English literature are used as a basis.

The committee realizes that not everyone will be fully satisfied with the way we defined the symbols for membrane distillation. Nevertheless, we hope that the people working in the field of membrane distillation will use these symbols in their publications.

Use of symbols

Only those symbols are given which are most frequently used in membrane distillation literature. The use of these symbols is strongly recommended. 


\section{Latin symbols}

$\begin{array}{lll}A & \text { Membrane area } & \mathrm{m}^{2} \\ C & \text { Concentration } & \mathrm{kg} / \mathrm{m}^{3} \\ \mathrm{CF} & \text { Concentration factor } & - \\ C_{\mathrm{p}} & \text { Heat capacity } & \mathrm{J} / \mathrm{kg} \mathrm{K} \\ D & \text { Diffusion coefficient } & \mathrm{m} / \\ d & \text { Pore size } & \mathrm{m} \\ \mathrm{EE} & \text { Evaporation efficiency } & - \\ \Delta H_{\mathrm{c}} & \text { Latent heat of condensation } & \mathrm{J} / \mathrm{kg} \\ \Delta H_{\text {vap }} & \text { Latent heat of vaporization } & \mathrm{J} / \mathrm{kg} \\ h & \text { Heat transfer coefficient } & \mathrm{W} / \mathrm{m}^{2} \mathrm{~K} \\ J & \text { Flux } & \\ & \text { mass flux } & \mathrm{kg} / \mathrm{m}^{2} \mathrm{~s} \\ & \text { molar flux } & \mathrm{kmol} / \mathrm{m}^{2} \mathrm{~s} \\ & \text { volume flux } & \mathrm{m} / \mathrm{m}^{2} \mathrm{~s} \\ & \text { gas flux } & \mathrm{Nm} / \mathrm{m}^{2} \mathrm{~s} \\ k & \text { Thermal conductivity } & \mathrm{W} / \mathrm{m} \mathrm{K}^{-} \\ \mathrm{LEP} & \text { Liquid entry pressure } & \mathrm{Pa} \\ l & \text { Thickness of the air gap } & \mathrm{m} \\ M & \text { Molecular weight } & \mathrm{Dalton} \\ P & \text { Pressure } & \mathrm{Pa} \\ \mathrm{PE} & \text { Process efficiency } & - \\ Q & \text { Heat transfer rate } & \mathrm{W} \\ Q^{\prime \prime} & \text { Heat flux } & \mathrm{W} / \mathrm{m}^{2} \\ R & \text { Retention coefficient } & - \\ R & \text { Universal gas constant } & \mathrm{J} / \mathrm{mol} \mathrm{K}^{2} \\ T & \text { Temperature } & \mathrm{K} \\ T & \text { Temp } & -\end{array}$

TPC Temperature polarization coefficient $t$ Time $\mathrm{s}$

$U \quad$ Overall heat transfer coefficient $\quad \mathrm{W} / \mathrm{m}^{2} \mathrm{~K}$

$u$ Cross-flow velocity $\mathrm{m} / \mathrm{s}$

$V \quad$ Volume $\mathrm{m}^{3}$

$w \quad$ Weight fraction -

$x \quad$ Molar fraction -

Greek symbols

$\begin{array}{lll}\alpha & \text { Selectivity } & - \\ \gamma & \text { Surface tension } & \mathrm{N} / \mathrm{m} \\ \Delta & \text { Difference } & - \\ \delta & \text { Membrane thickness } & \mu \mathrm{m} \\ \epsilon & \text { Membrane porosity } & - \\ \eta & \text { Gas viscosity } & \mathrm{Pa} \mathrm{s} \\ \mu & \text { Liquid viscosity } & \mathrm{Pa} \mathrm{s}\end{array}$




$\begin{array}{lll}\pi & \text { Osmotic pressure } & \mathrm{Pa} \\ \rho & \text { Density } & \mathrm{kg} / \mathrm{m}^{3} \\ \chi & \text { Membrane pore tortuosity } & -\end{array}$

\begin{tabular}{ll}
\multicolumn{2}{c}{ Subscripts } \\
av & Average \\
b & Bulk \\
f & Feed \\
i,j & Index \\
l & Liquid \\
m & Membrane \\
o & Initial, zero \\
p & Permeate \\
s & Solute \\
$t$ & Time \\
v & Vapour \\
w & Water
\end{tabular}

Use of units

A general rule for the use of units is, that the unit should be as standard as possible. This means that in principle SI-units or related units should be used. For instance, the membrane thickness can be given in micrometers instead of meters.

The units which are recommended are given in the list of symbols.

\section{REFERENCES}

1 V. Gekas, Desalination, 68 (1988) 77.

2 P. Aptel et al., J. Membr. Sci., 1 (1976) 271.

3 K. Schneider and T.J. van Gassel, Chem. Ing. Tech., 56 (1984) 514.

4 Swedish National Development Company, prospect about membrane distillation.

5 W.L. Gore et al., Eur. Pat., EP 88315.

6 I. Cabasso et al., J. Appl. Polym. Sci., 18 (1974) 2117.

7 R.A. Cotton et al., in B.J. Dutka (Editor), Membrane Filtration; Application, Techniques and Problems, Marcel Dekker, New York, Basel, pp. 19-39.

\section{APPENDIX}

\section{Determination of membrane characteristics}

Porosity of the membrane

The porosity of the membrane is defined as the volume of the pores divided by the total volume of the membrane. The porosity can be measured by making 
use of a pyknometer, a balance, isopropyl alcohol (IPA) and water. In this method use is made of the fact that IPA penetrates into the pores of the membrane and water does not penetrate into the pores of the membrane.

First, the density of the polymer material is calculated using the following formula:

$\rho_{\mathrm{pol}}=\frac{\rho_{\mathrm{IPA}} \mathrm{wt} .3}{\mathrm{wt} .1+\mathrm{wt} .3-\mathrm{wt} .2}$

in which wt.1 = weight of the pyknometer with IPA; wt.2= weight of the pyknometer with IPA and membrane; wt. $3=$ dry weight of the membrane. In the same way the density of the membrane can be calculated according to the following formula:

$\rho_{\mathrm{m}}=\frac{\rho_{\mathrm{w}} \mathrm{wt} .3}{\mathrm{wt} .1+\mathrm{wt} .3-\mathrm{wt} .2}$

in which wt. $1=$ weight of the pyknometer with water; wt. $2=$ weight of the pyknometer with water and membrane; wt. $3=$ dry weight of the membrane.

The porosity of the membrane can be calculated by the following formula:

$\epsilon=1-\frac{\rho_{\mathrm{m}}}{\rho_{\mathrm{pol}}}$

\section{Determination of $L E P_{\mathrm{w}}$}

The following procedure is suggested for the determination of the liquidentry-pressure of water. The apparatus for this measurement is shown in Fig. 5.

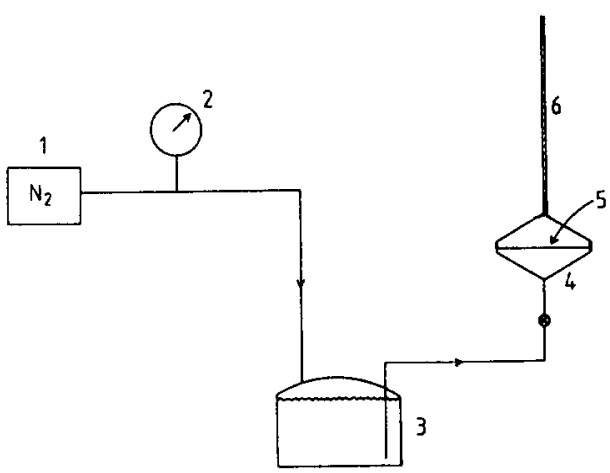

Fig. 5. Apparatus for the determination of the liquid-entry-pressure. $1=$ Gas cylinder with nitro gen; $2=$ manometer; $3=$ liquid feed; $4=$ measuring cell; $5=$ membrane; $6=$ measuring pipette. 
Measuring procedure (Fig. 5)

The dry hydrophobic membrane 5 is placed into the measuring cell 4 and the reservoir 3 is filled with the liquid feed mixture (in this case water). The half cell which forms the permeate side of the membrane is also filled water. By means of a gas cylinder, filled with nitrogen, a slight pressure is applied to the system in order to remove all the gas at the feed side of the membrane. The pressure which is used to remove the gas should of course be lower than the liquid-entry-pressure. During the degasification of the feed a continuous stream of gas bubbles passes through the membrane. As soon as this stream of gas bubbles stops, there is no more gas in the feed compartment. After the degasification of the permeate side the measurement can be started.

During the measurement the pressure is raised stepwise (with 0.1 bar). At each installed pressure one should watch whether a flux through the membrane occurs. The minimum pressure at which a (continuous) flux is observed is called "liquid-entry-pressure". 